\title{
Design and status of the NGS WFS of MAORY
}

\author{
Marco Bonaglia $^{a}$, Guido Agapito $^{a}$, Lorenzo Busoni $^{a}$, Paolo Ciliegi $^{b}$, Fausto Cortecchia $^{b}$, \\ Emiliano Diolaiti ${ }^{b}$, Gianluca Di Rico $^{c}$, Simone Esposito ${ }^{a}$, Christophe Giordano ${ }^{a}$, Cedric \\ Plantet $^{a}$, Armando Riccardi ${ }^{a}$. \\ ${ }^{a}$ Osservatorio Astrofisico di Arcetri, l.go E. Fermi 5, 50125 Firenze, Italy; \\ ${ }^{b}$ Osservatorio Astronomico di Bologna, v. P. Gobetti 93, 40129 Bologna, Italy; \\ ${ }^{c}$ Osservatorio Astronomico di Teramo, v. M. Maggini, 64100 Teramo, Italy.
}

\begin{abstract}
MAORY is the first-light multi-conjugate AO facility of the E-ELT, providing a $>30 \%$ Strehl ratio (evaluated in $\mathrm{K}$ band, median seeing conditions) over the MICADO $53 \times 53$ arcsec FoV and ensuring an overall sky coverage of 50\%. MAORY will implement 3 NGS WFS having a double functionality: sensing the atmospheric low-order modes (LO-WFS) and de-trending the LGS WFS measurements (Ref-WFS).

To maximize the AO sky-coverage the preliminary design of the LO-WFS foresees a $2 \times 2$ subapertures ShackHartmann sensor working in the $\mathrm{H}$ band, where the partial AO-correction regime and the low-noise detectors based on APD technology will enhance the WFS sensitivity. The Reference WFS will measure at slow rate $(1 \div 10 \mathrm{~s})$ the first $\sim 100$ modes of the atmospheric aberration and it will correct the LGS measurements that are affected by the Sodium layer drifts. The Ref-WFS will be a $10 \times 10 \mathrm{SH}$ making use of a CCD220-based detector. Hence it will work at visibile wavelenghts, using the same NGS of the LO-WFS.

We present here the results of a study aimed to find the best design solutions for the MAORY NGS WFS in preparation of the project preliminary design review of scheduled in February 2018. We describe first the output of numerical simulations to find the best compromise between the working bandwidth and sampling of the LO-WFS and the sky-coverage corresponding to the expected performance. Then we describe the arrangement for the NGS WFS module, hosted on top of the MICADO cryostat, analyzing the static and dynamic properties of the NGS module structure. Finally we present the opto-mechanical layout for the NGS WFS and its degrees of freedom.
\end{abstract}

Keywords: European Extremely Large Telescope, multi-conjugated adaptive optics, Shack-Hartmann wavefront sensor, end-to-end simulations, sky coverage.

\section{INTRODUCTION}

MAORY will be the multi-conjugate adaptive optics (MCAO) instrument of the E-ELT. ${ }^{1}$ It will provide a $30 \%$ Strehl ratio (SR) (50\% goal) in K band under median seeing conditions $(\sim 0.8$ arcsec seeing), ensuring a $<10 \%$ $\mathrm{SR}$ variation across the scientific field-of-view (FoV). MAORY will primarly serve MICADO, ${ }^{2}$ the E-ELT nearinfrared imager and spectrograph having a $53 \times 53$ arcsec FoV, from its first light. The instrument is targeted to perform astrometric and deep photometry observations with very high relative accuracy and the goal of MAORY is to ensure a $50 \%$ sky-coverage with MCAO for these science programs.

The baseline design of MAORY relies on:

- 2 deformable mirrors: the E-ELT M4 ${ }^{3}$ as ground-layer corrector and a post-focal DM conjugated approximately to $12 \mathrm{~km}$ altitude as high-layer corrector*,

- up to 6 Sodium laser guide stars (LGS) distributed within 1 arcmin radius and the corresponding wavefront sensors (WFS) to perform a tomographic measurement of the atmospheric turbulence,

Send correspondence to: Marco Bonaglia, E-mail: mbona@arcetri.astro.it, Telephone: +39 0552752200

*MAORY foresees the insertion of a second post-focal DM as possible upgrade path: it will be conjugated to $\sim 4 \mathrm{~km}$ altitude and in the baseline design it is replaced by a rigid mirror with the same optical properties. 
- 3 natural guide star (NGS) WFS to complete LGS WFS measurements with the low-order modes affected by the atmospheric anisoplanatism and to de-trend the higher-order LGS measurements affected by the Sodium layer instabilities.

The double functionality of the NGS WFS is implemented by providing two optical paths on the same board: a low-order (LO) and a reference (Ref) WFS. Each NGS WFS will be placed on a pair of acquisition stages in order to patrol the 1.5 arcmin radius that represents the NGS acquisition area.

A preliminary study based on numerical simulations carried out in Arcetri ${ }^{6}$ has shown that the best performance for the LO-WFS, both in terms of sensitivity and limiting magnitude, are reached with a $2 \times 2$ subapertures Shack-Hartmann sensor (SHS) working in the $\mathrm{H}$ band with a frame rate of about $1 \mathrm{kHz}$. The best layout for the reference WFS is a $10 \times 10$ subapertures SHS making use of the optical wavelengths of the same NGS. The Ref-WFS has a frame rate of $0.1 \div 1.0 \mathrm{~Hz}$ and it is interfaced to the MAORY real-time computer through the instrument control software. ${ }^{5}$ Section 2 completes the previous performance analysis also computing the corresponding sky coverage and studying its evolution with different set of design parameters.

Figure 1 shows a model of the E-ELT Nasmyth platform highlighting the space allocation for the main components of MAORY and MICADO. The optical layout of the MCAO post focal relay ${ }^{4}$ is also shown.
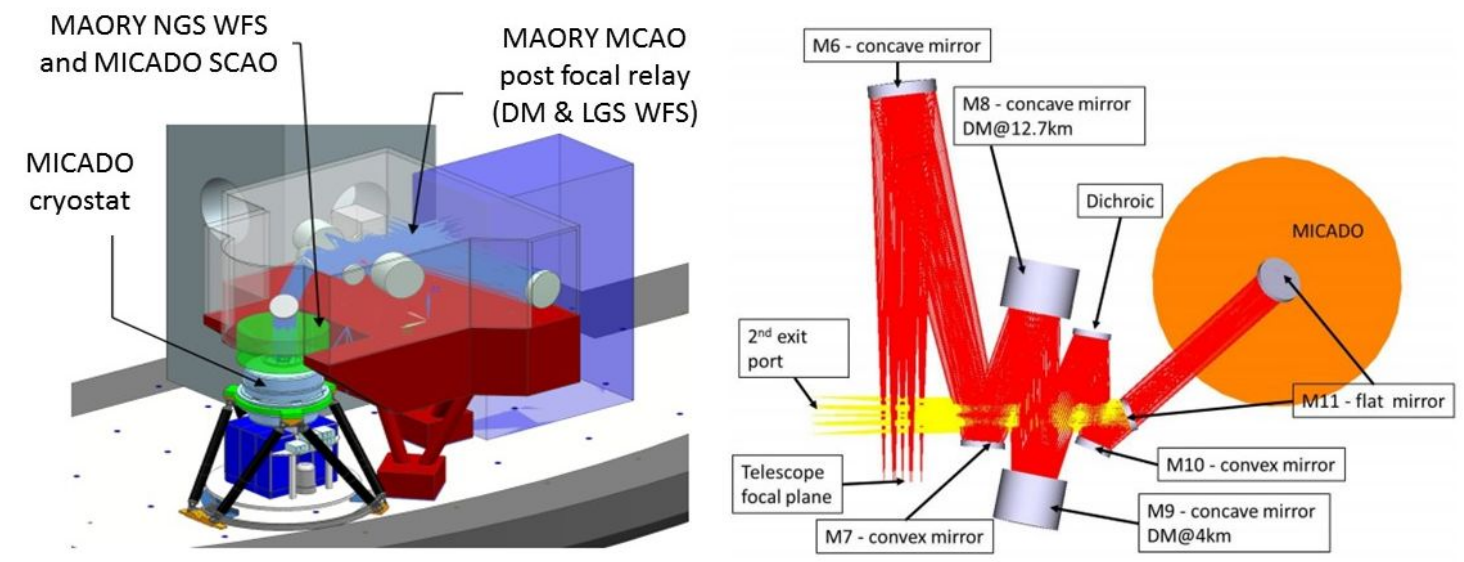

Figure 1. Left: model of the E-ELT Nasmyth platform showing the MAORY post focal MCAO realy bench (red color), the MICADO instrument assembly and the position of a possible second generation instrument (blue volume). The green color highlights the volume where both the MAORY NGS WFS and the MICADO SCAO system will be installed. Right: optical layout of the MCAO post focal ralay highlighting the conjugation altitudes of the DMs and the exit focal planes.

The 3 NGS WFS of MAORY are placed close to the exit focal plane of the MCAO relay within a volume shared with the instrument MICADO (highlighted in green color in Figure 1). The field de-rotation is provided by the MICADO de-rotator that also supports the whole NGS WFS module. A description of the NGS WFS mechanical structure and its static and dynamic perfomance is given in Section 3 while the optical design of the NGS WFS and its degrees of freedom are presented in Section 4.

\section{PERFORMANCE AND SKY COVERAGE}

In order to validate the choice of the pupil sampling adopted in the WFS design, we compare the sky coverage obtained for a SHS working in the $\mathrm{H}$ band and having between 2 and 5 subapertures on the pupil diameter. In order to do this, we define a minimum flux for each sampling and then we check the statistical availability of NGS within the FoV according to a star count model. ${ }^{9}$ 


\subsection{Limiting flux definition}

The limiting flux depends on the requirement of AO wavefront residual. The wavefront residual variance $\sigma_{W F R}^{2}$ can be written as:

$$
\sigma_{W F R}^{2}=\sigma_{H O}^{2}+\sigma_{(L O, r e c)}^{2}+\sigma_{(L O, n o i s e)}^{2},
$$

where $\sigma_{H O}^{2}$ is the residual variance from the high-order correction. This is defined by the level of MCAO pre-correction assumed to simulate the partial AO correction expected in correspondence of the NGS patrol field. $\sigma_{(L O, r e c)}^{2}$ is the residual variance from the low-order correction at high flux (negligible noise error) and $\sigma_{(L O, n o i s e)}^{2}$ is the low-order residual variance due to noise. The first two terms depend on the WFS line of sight while $\sigma_{(L O, n o i s e)}^{2}$ is the only term that evolves with the NGS magnitude, hence we must focus to analyze the behavior of this term.

The $\sigma_{(L O, \text { noise })}^{2}$ is extimted from the LO-WFS performance analysis carried out in Arcetri using the PAS$\mathrm{SATA}^{7}$ numerical simulation code. The input for the end-to-end simulations were the MCAO-precorrected phase screens provided by ESO ${ }^{8}$ other simulation parameters were:

- seeing: 0.64 arcsec (ESO median seeing condition)

- Zenith angle: $30^{\circ}$

- 6 LGS asterism at 1 arcmin radius

- 3 NGS at 70 arcsec at the vertexes of an equilateral triangle

- 5 independent realizations of 500 frames each at $500 \mathrm{~Hz}$ rate

The numerical simulations output are the estimated low-order residuals (tip/tilt, focus and astigmatisms) in function of different pupil sampling for the SHS: $2 \times 2,3 \times 3,4 \times 4$ or $5 \times 5$ subapertures. The plot in Figure 2 summarizes the results obtained: at high flux, the LO residual is high because of aliasing effects. The way these aliasing effects act on the residual is not trivial and still under study. At low flux, there is a clear advantage in using the $2 \times 2$, as it could be expected (better signal-to-noise ratio compared to other pupil sampling). The LO residual budget is not fixed yet, but should be in the range $150-250 \mathrm{~nm}$. This corresponds to have a limiting magnitudes of $18-19$ in $\mathrm{H}$ band for the $2 \times 2$ SHS.

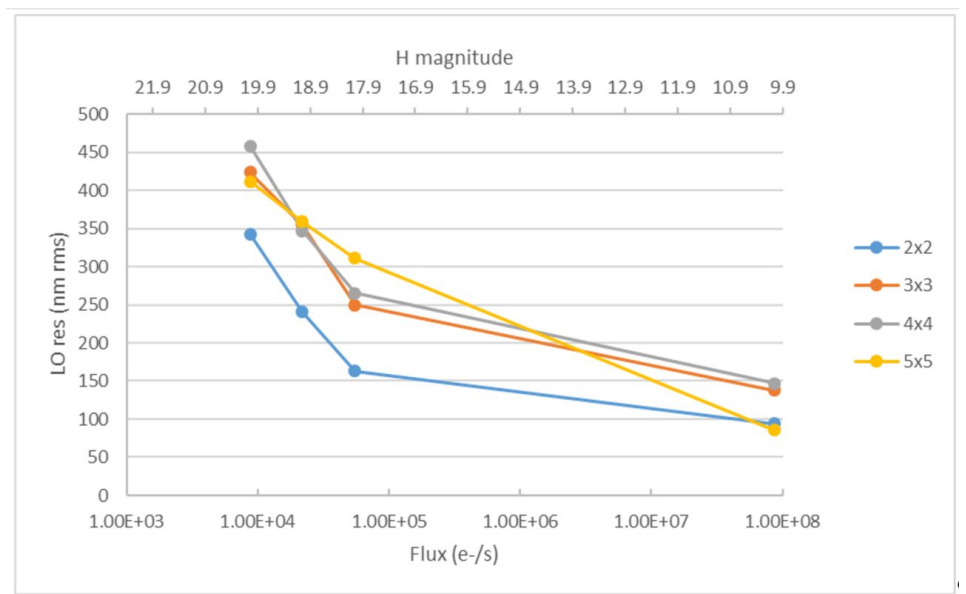

Figure 2. LO residuals averaged over the 3 NGS and the 5 turbulence occurrences as a function of incoming flux for the different SHS configurations. 


\subsection{Sky coverage analysis}

Having defined the limiting flux for each pupil sampling, we have to check the availability of stars within the annular FoV that is allocated to each NGS WFS. The sky coverage is given by the probability to have 3 or more stars of magnitude brighter than the limiting flux within the annular FoV shown in Figure 3. The FoV inner radius is $40 \operatorname{arcsec}$ (corresponding to the inscribed $53 \times 53 \operatorname{arcsec}$ FoV of MICADO) and the outer radius is 90 arcsec. The sky coverage analysis also accounts for the finite dimension of the NGS WFS pickoff mirror (POM) arm (highlighted in yellow color in Figure 3).
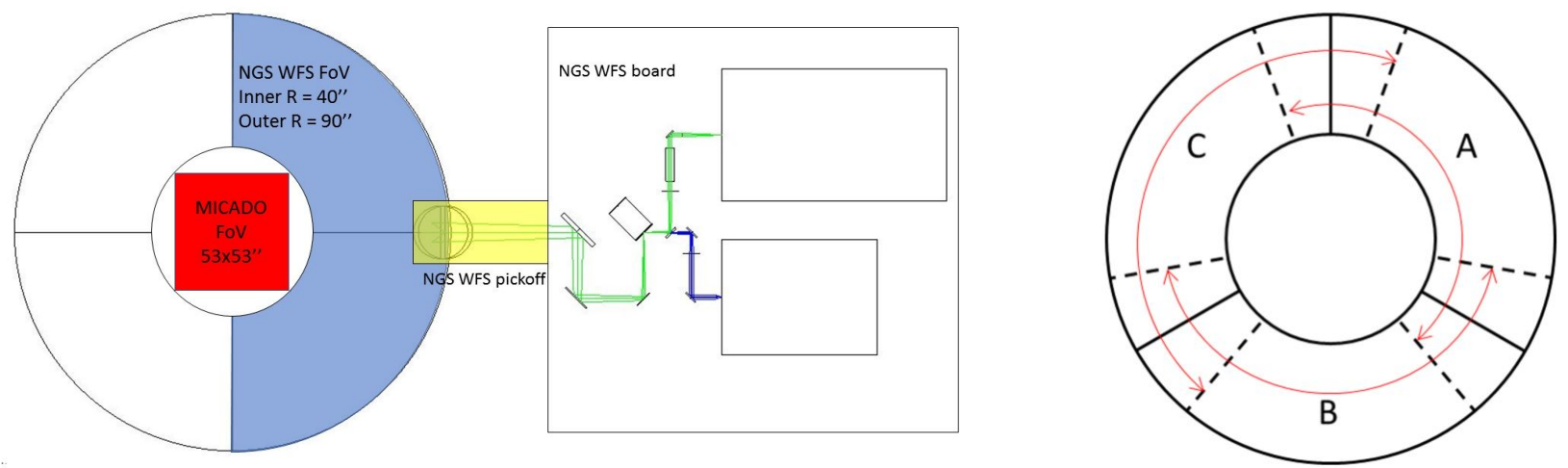

Figure 3. Left: scheme of the NGS WFS FoV (blue area) considered in the scky coverage analysis. The yellow color highlights the profile of the NGS WFS pickoff arm that will limit the possibility for 2 different WFS to reach a pair of NGS that are too close each other. Right: scheme of the FoVs for the 3 NGS WFS and of their overlap angle, represented by the sectors delimited by the dashed lines.

In this analysis we rely on the TRILEGAL galaxy model, ${ }^{9}$ using the default configuration parameters of version 1.6, to retrieve the number of stars available in a FoV of $3 \times 3^{\circ}$ at the north galactic pole. Then, the NGS WSF FoV is randomly centered on the stars distribution and we check if the stars' configuration is valid or not. This operation is repeated $10^{5}$ times to create a sufficient statistics. A stars' configuration is declared valid if:

1. each NGS WFS can reach at least 1 star brighter than the selected limiting magnitude.

2. the distance between 2 stars must be greater than the pickoff mirror profile, to avoid collision.

3. the POM should not vignette MICADO's FoV, meaning that the distance of the selected star to the center minus the POM's radius should be greater than 40 arcsec.

The procedure we used to verify these 3 rules is resumed below. Make reference to Figure 4 for details.

- Register all stars under the limiting magnitude reachable by each sensor. Some can be counted twice because of the overlap. This is taken into account in the next steps.

- Remove stars that are too close to the center to respect the non-vignetting condition on MICADO's FoV.

- Compute all distances between stars reachable by neighboring sensors (distances between stars of sensors $\mathrm{A}$ and $\mathrm{B}$, sensors $\mathrm{A}$ and $\mathrm{C}$, and sensors $\mathrm{B}$ and $\mathrm{C}$ ).

- Verify that for each couple of sensors (A, B), (A, C), (B, C), there is at least one couple of stars that are distant from each other by more than the POM diameter. If a couple of stars is actually twice the same star, the distance is 0 , and the couple is discarded. 

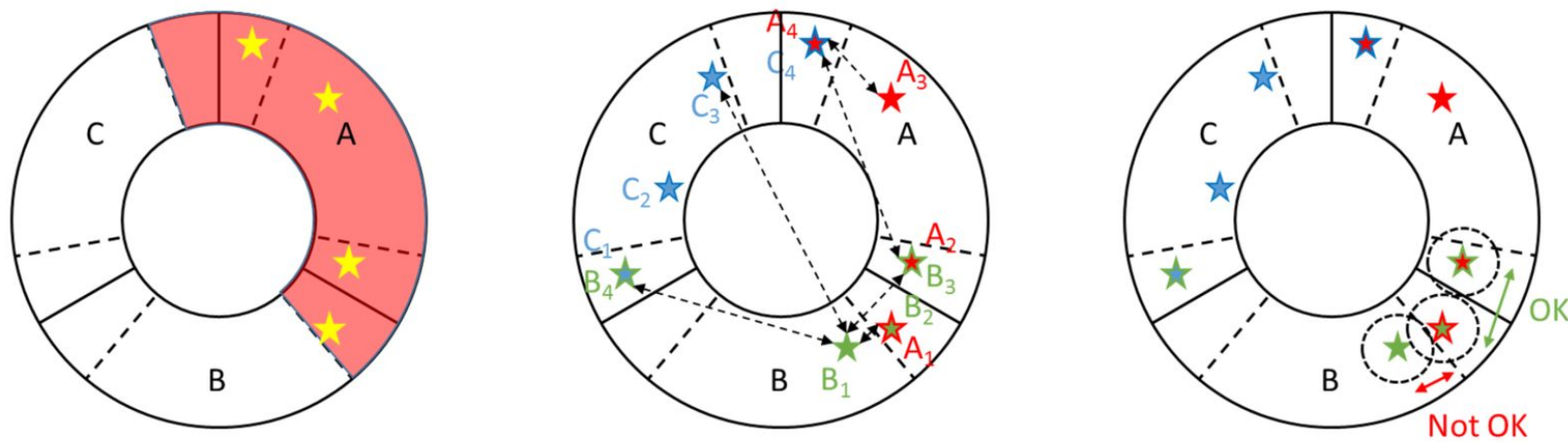

Figure 4. Scheme of the method we used to detect a valid NGS configuration. Left: register all stars under the limiting magnitude reachable by each sensor (remove those too close to the center to avoid vignetting). Center: compute distances between the registered stars. Right: check there is at least 1 couple of stars distant enough from each other (more than the pick-off mirror diameter) for each couple of sensors.

Having an overlap between the WFS dedicated areas helps to improve the sky coverage. The impact of the overlap angle for different limiting magnitudes is plotted in Figure 5. In this analysis we assume that the POM is infinitely small. Obviously the sky coverage increases more quickly for low overlap angles, and it saturates to the upper limit when going towards higher angles. The impact is quite significant: for a limiting magnitude $\mathrm{H}$ $=19$, the sky coverage is $40 \%$ without overlap and almost $60 \%$ with a $20^{\circ}$ overlap.

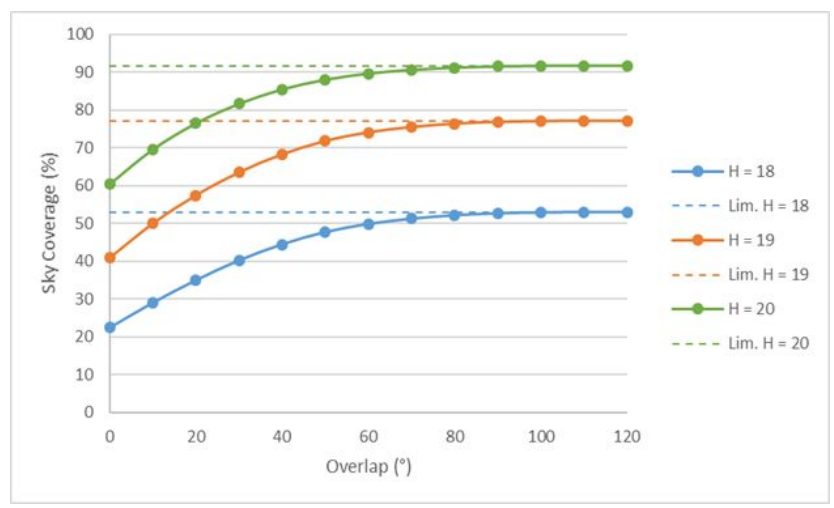

Figure 5. Sky coverage as a function of the overlap angle and limiting magnitude (colors), assuming the pickoff mirror is infinitely small. An overlap of $120^{\circ}$ means that all sensors have access to the whole patrol field. The dotted lines represent the upper limit for the sky coverage, i. e. the probability of having 3 stars anywhere in the patrol field.

The impact of the finite POM size on the sky coverage has to be taken into account, as it lowers the asterism possibilities (we must avoid POMs collisions and vignetting on MICADO's FoV). Figure 6 shows the evolution of sky coverage as a function of the POMs diameter without overlap or with an overlap of $30^{\circ}$ (baseline design for the NGS WFS). The POM has a diameter of $60 \mathrm{~mm}$ (eqivalent to approximately 18 arcsec on-sky). This means the limiting magnitude should be slightly lower than 19 to fulfill the requirement of a $50 \%$ sky coverage. The results presented in section 2.1 show that this magnitude could be sufficient, depending on the needed residual on the low orders. The sky coverage corresponding to these results is between $33 \%$ and $55 \%$ (see magnitudes 18 and 19 in Figure 6 right). In any case, decreasing the POM size will not improve significantly the sky coverage: the minimum size is 7.6 arcsec (footprint of the on-axis beam), we thus cannot expect to go lower than approximately 10 arcsec, which represents a gain of a few percents only on the sky coverage. 


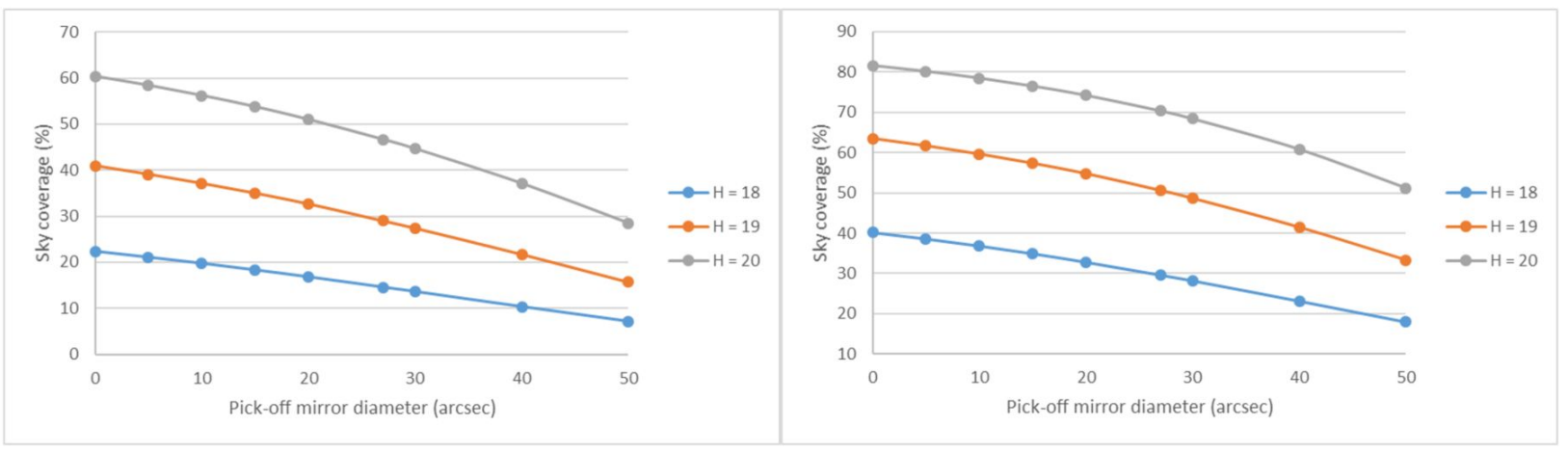

Figure 6. Sky coverage evolution with the pick-off mirror diameter without overlap (left) and with an overlap of $30^{\circ}$ (right). The baseline design for the NGS WFS has a POM dimension equivalent to 27 arcsec on-sky.

\section{NGS MODULE}

MAORY will implement the 3 NGS WFS within a volume placed between the MCAO relay bench and the MICADO cryostat, highlighted in green color in Figure 1. This volume measures $2.6 \mathrm{~m}$ in diameter and $1.0 \mathrm{~m}$ in height and it is shared with MICADO. The volume has been split vertically in 2 parts: the lower one $(0.6$ $\mathrm{m}$ in height) is dedicated to the MAORY NGS WFS, the upper one $(0.4 \mathrm{~m}$ in height) is where the MICADO SCAO system will be implemented. ${ }^{10}$ The common interface for the 2 WFS systems is represented by a support structure (red color in Figure 7) directly supported by the MICADO rotator and placed $200 \mathrm{~mm}$ before the MAORY exit focal plane. Two different benches will support respectively the MICADO SCAO (black color in Figure 7) and the MAORY NGS WFS systems (blue color).
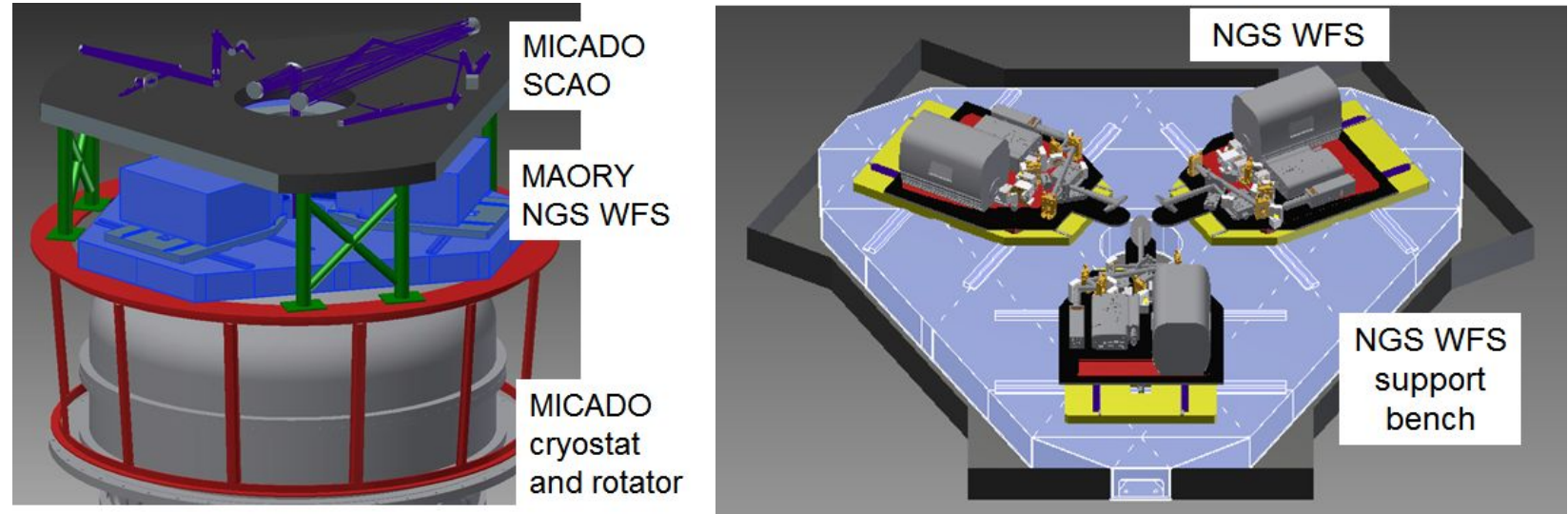

Figure 7. Left: model of the MAORY NGS module (blue color) and of the common support structure (red) attached to the MICADO rotator and cryostat (light gray). The MICADO SCAO system will be installed on the upper bench (dark gray). Right: detail of the NGS WFS module highlithing the WFS support bench (light blue) and the 3 NGS WFS, each one mounted on a pair of linear stages (yellow and red) needed for the NGS acquisition.

The NGS WFS module will implement:

1. A $150 \mathrm{~mm}$ thick steel-honeycomb plate to support the 3 NGS WFS, detailed in Section 3.1.

2. 3 pairs of acquisition stages to allow the NGS WFS to patrol the FoV schematized in Figure 3.

3. A lateral cover to preserve the thermally controlled environment of the MCAO bench. 
4. 2 electronics cabinets, hosted in the MICADO co-rotating structure below the cryostat, detailed in Section 3.2 .

\subsection{NGS WFS bench}

A feasibility study for the NGS WFS support bench has been outsourced to Tomelleri S.r.l.. The bench is made out of a steel honeycomb structure: the top plate is $6 \mathrm{~mm}$ thick, the bottom one is $4 \mathrm{~mm}$ thick and the distance between the 2 plates is $150 \mathrm{~mm}$. The total mass of the bench is about $380 \mathrm{~kg}$. The interface between the NGS WFS and the bench is represented by the tangential rails of the acquisition stages. Indeed those rails will be directly welded to the bench top plate in correspondence of the vertical stiffening structures as shown in Figure 7. The interface between the bench and the support structure is represented by 3 triangular pods placed in correspondence of the stiffening elements of the honeycomb, as close as possible to the NGS WFS nominal working position.

For servicing purposes each NGS WFS can be easily removed from the GD side. It is possible to perform this operation by fully retracting each WFS board along the radial rails and then attaching the board to the telescope crane. In case the complete NGS module requires servicing the bench will be made fully accessible by first craning out the MICADO SCAO system. Another possibility is to detach the NGS module from the support structure, crane it out and then exchange it with a dummy bench to preserve the load distribution on the instrument rotator. This will allow to re-install quickly the MICADO SCAO system minimizing down time.

The NGS WFS support bench behavior has been studied both in static and dynamic conditions. In both cases the NGS WFS payload has been simulated adding three $100 \mathrm{~kg}$ masses in compression on top of the tangential rails. Each mass is applied on 4 small points to simulate for the rail carriers effects.

The static analysis shows the deformation of the NGS WFS bench when the payload of the 3 WFS is moved along the rails. Alternatively the 3 payloads have been offsetted by $60 \mathrm{~mm}$ in the tangential and radial directions and the differential flexure of the bench surface has been measured. From the position of the 4 points corresponding to the rail carries it has been possible to evaluate the induced displacement of the NGS WFS board. The maximum displacement in the bench plane amounts to $(\Delta x, \Delta y)=0.5 \mu \mathrm{m}$ while the maximum pitch and yaw is $(\Delta \phi, \Delta \theta)=0.56$ arcsec. These values are in agreement with the stability requirement imposed by the MAORY astrometric error budget, that allocated 1.8 mas ( $\sim \mu \mathrm{m}$ on the FP) to the NGS WFS positioning error.

The dynamic analysis has been performed to determine the first eigenfrequencies for the NGS module. The NGS WFS bench has been constrained in correspondence of the 3 interface pads. Figure 8 shows the first 3 modes measured at 96, 154 and $155 \mathrm{~Hz}$ respectively, more than 3 times bigger than the first mode expected from the MICADO structure $(\sim 30 \mathrm{~Hz}) .{ }^{11}$

\subsection{NGS WFS control electronics}

All the control electronics of the MAORY NGS module will be placed inside two of the six cabinets on the MICADO co-rotating platform, below the octopod structure supporting both the NGS module and the MICADO cryostat as shown in Figure 9. The platform follows the field de-rotator for the full $\pm 270^{\circ}$ with an accuracy of $\pm 1^{\circ}$. This solution allows to avoid the use of a cable-wrap for the connections between the 3 NGS WFS and the electronics cabinets underneath minimizing the cables length. The steady central flange around the MICADO cryostat provides feed-through for electrical cables, optical fibers and glycol-water cooling pipes to the cabinets, while a backward-bending cable-wrap all around the co-rotating platform is used for the connection to the observatory Service Connection Points (SCPs).

The two thermal controlled cabinets will provide 30 RU each to settle the full NGS module Instrument Control Electronics (ICE). This will be based on Beckhoff EtherCAT PLCs, according to the ESO specifications for the design of instrument electronics. ${ }^{12}$ Through the EtherCAT fieldbus it is possible to operate all the function terminals which provide:

- motion control for DC brush and stepper motors,

- analog and digital I/O (for continuous and discrete control and monitoring of devices, alarms and warnings), 


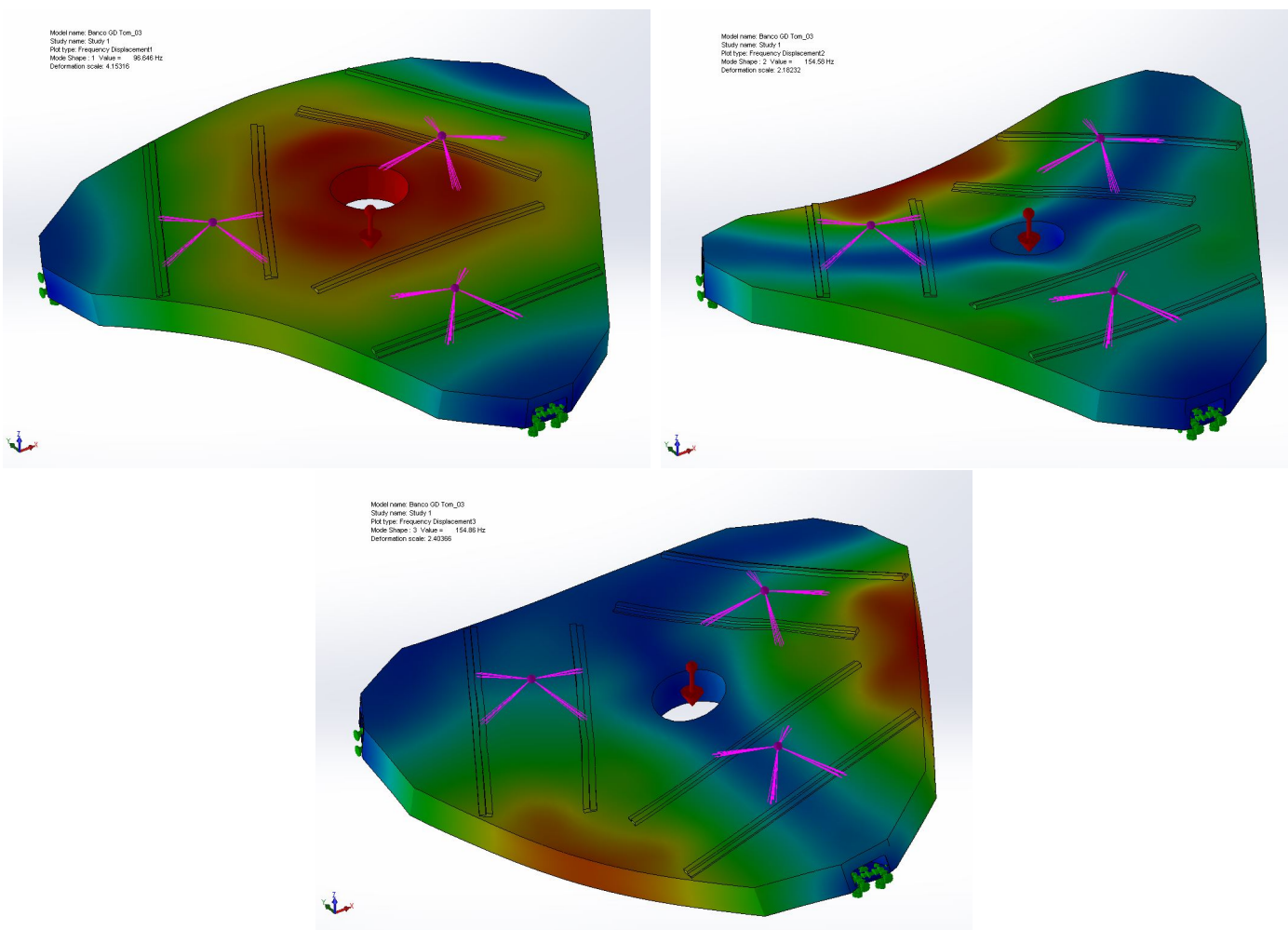

Figure 8. Results obtained in the FEA analysis of the NGS WFS support bench. The first 3 eigenfrequencies measured are 96,154 and $155 \mathrm{~Hz}$.
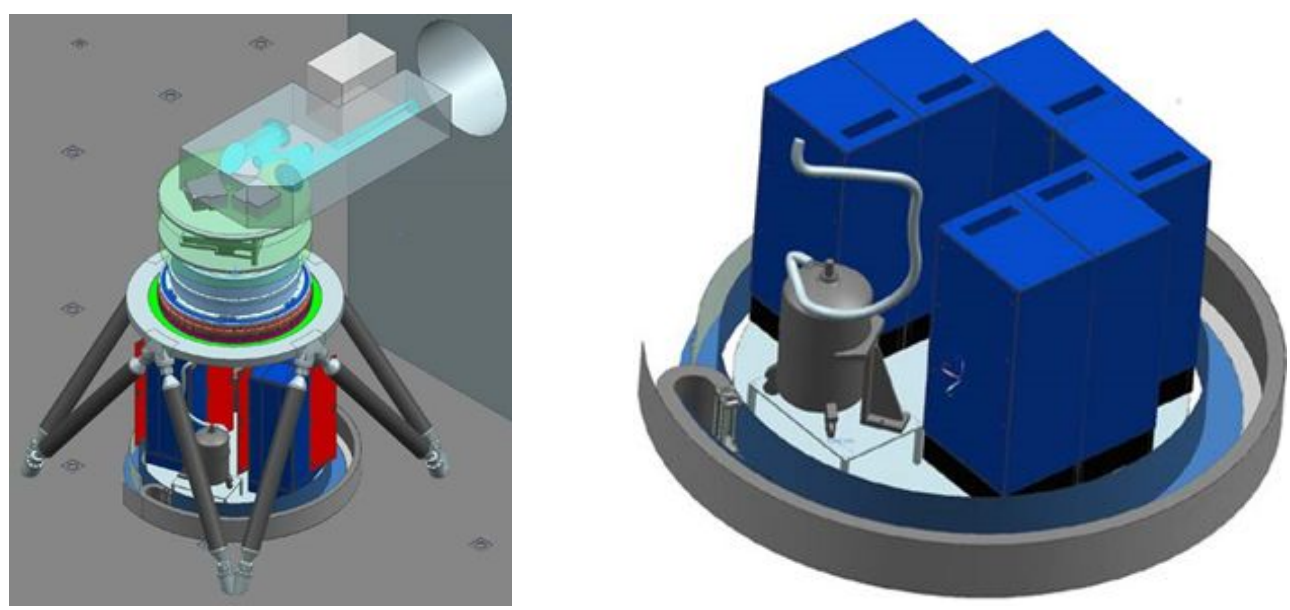

Figure 9. Left: model of the octopod structure supporting the MICADO cryostat, the SCAO system and the MAORY NGS module. Right: detail of the co-rotating platform below the cryostat. The platform will host six cabinets used for the MICADO and MAORY NGS Control Electronics.

- ethernet and serial interface for communication.

Brushless motors (BLDC) stages can be controlled through Beckhoff or third party EtherCAT servo-drives. All the motion control and I/O functions relays on the Beckhoff TwinCAT NC software layer which provide a 
standard software interface to the PLC software thorough the PLCopen MC compliant library.

Each NGS WFS has six motorized functions, a piezo TT mirror and two WFS cameras (for the visible and the infrared channel), as detailed in Section 4. Commercial off-the-shelf devices have been selected for most of the electronics components (motorized stages, power supplies, PLCs, terminals, servo drives and piezo controllers), while custom two axes linear stages will be needed to move the whole WFS board on the focal plane for the NGS acquisition. ESO will provide the three visible CCD detectors and the three IR cameras together with their readout and control electronics. All the ICE will be mounted on standard 19-inches racks inside the 2 NGS module cabinets, as shown in Figure 10. This solution ensures an easy dismounting and a better accessibility during the MAIT phase and for on telescope maintenance.
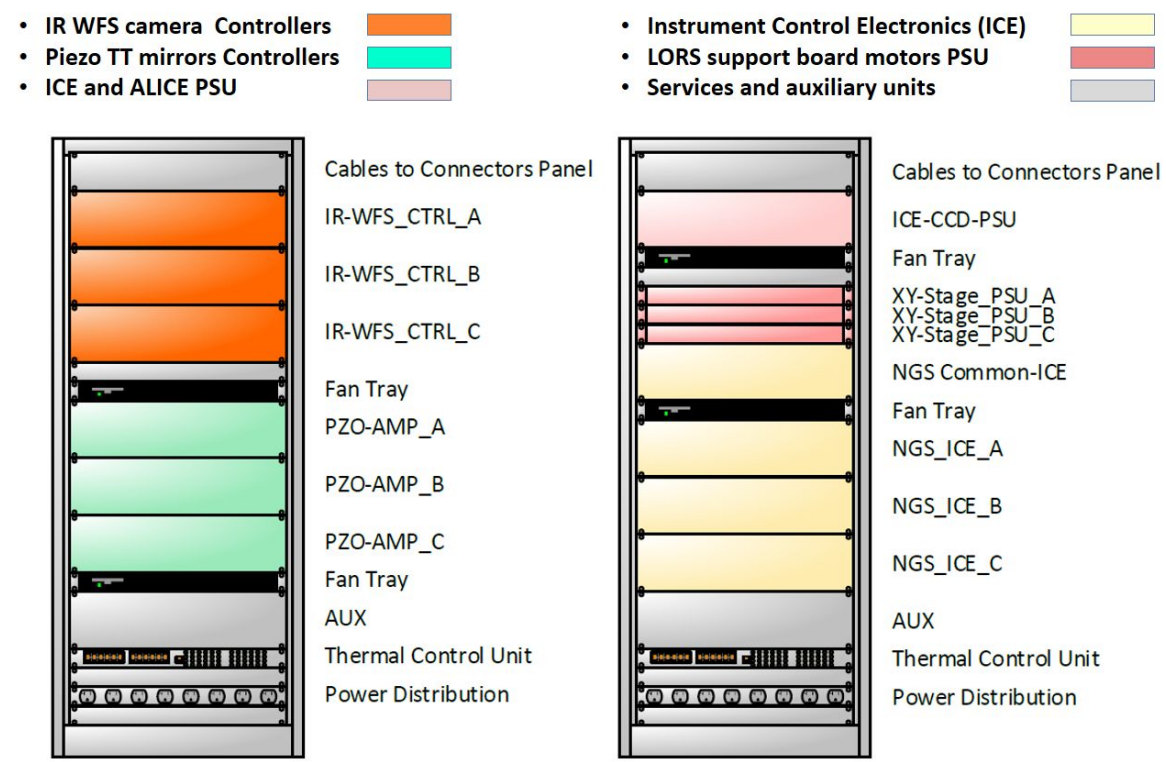

Figure 10. Distribution of the NGS WFS control electronics within the 2 cabinets hosted on the MICADO co-rotating structure. Power supplies and controllers for the infrared and visible cameras are hosted in the top part of both racks: they are highligthed in orange and pink colors respectively. Controllers and amplifiers for the piezo stages are highlighted in green, the XY linear stages controllers are red and the Beckoff ICE are yellow.

\section{NGS WFS DESIGN}

The baseline for the design of the MAORY NGS WFS is to have 3 identical units, each one able to pickoff an NGS within a portion of the total FoV. The 3 units will be disposed at $120^{\circ}$ geometry around the MICADO FoV and they will acquire the NGS light through a pickoff mirror (POM). Figure 11 schematizes the NGS WFS pickoff scheme. The NGS light will be picked off at $500 \mathrm{~mm}$ distance from the output focal plane of MAORY and it will be folded by the POM into a plane parallel to the NGS WFS support bench and to the rotator plane. The whole NGS WFS opto-mechanics will be mounted on a couple of orthogonal linear stages that will allow it to patrol the $660 \times 330 \mathrm{~mm}$ area designated to the NGS pickoff. The details of the NGS WFS patrol FoV are given in Section 2.2 .

As shown in Figure 11 the first optical element of the NGS WFS is the POM. This will be an elliptical mirror of 116 and $80 \mathrm{~mm}$ major and minor axes respectively and $10 \mathrm{~mm}$ thickness. These dimensions are required to fully reflect the LO and Ref WFS FoVs and to accommodate for an incoming beam tilt up to $\sim 2.6^{\circ}$, caused by the finite distance of the WFS input pupil. After the POM a fold mirror deflects the light to a reflective trombone mounted on a linear stage with $25 \mathrm{~mm}$ travel range (i.e. Newport M-VP-25X serie) to compensate for 
the differential focus between the 3 NGS WFS. The last element of the NGS WFS foreoptics is a pupil steering mirror mounted on a piezo-actuated tip-tilt platform (i.e. Physik Instrumente S335). This element is needed to adjust the pupil position on the LO and Ref-WFS lenslet arrays in such a way to compensate for flexures in the telescope and relay structures and to remove the incoming chief-ray tilt. This device will allow the LO and Ref WFSs optics to work on-axis hence to minimize the differential aberrations introduced by the field selection.
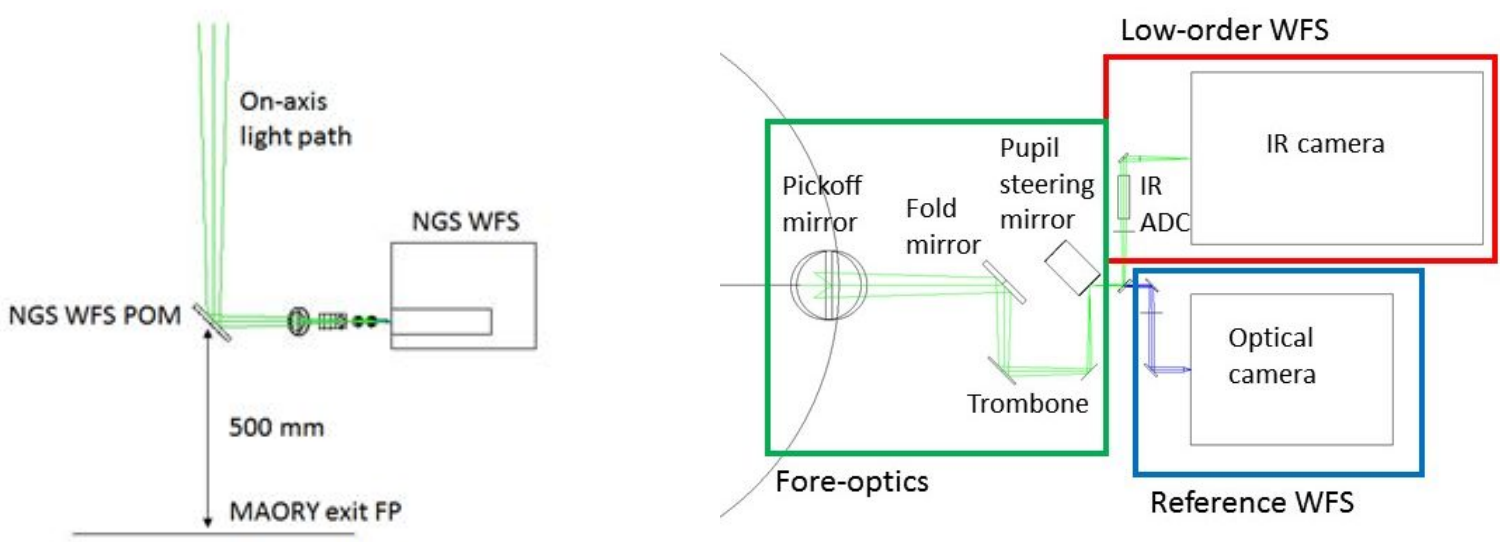

Figure 11. Scheme of the NGS pickoff from a single NGS WFS view from side (left) or from top (right). The distance between the POM center and the MAORY exit focal plane is $500 \mathrm{~mm}$. The optical layout of the NGS WFS is composed by 3 main blocks. 1) the first five elements (green box) are reflective optics used to compensate for differential optical paths introduced by field selection. 2) the red box highlights the low-order WFS optics, 3) the blue box highlights the reference WFS optics.

A dichroic will split the NGS light between the low-order and reference WFS: wavelengths $>1.1 \mu \mathrm{m}$ will be reflected towards the LO-WFS while wavelengths $<1.1 \mu \mathrm{m}$ will be transmitted to the Ref-WFS. The layout of the 2 WFS is similar: both optical system produce a $5.76 \mathrm{~mm}$ diameter pupil through a refractive collimator. The pupil image is sliced by a $2 \times 2$ micro-lenses on the LO-WFS and by $10 \times 10$ micro-lenses on the Reference WFS. In addition the LO WFS make use of an ADC to take advantage of the partially corrected PSF at infrared wavelengths. In the preliminary design of the NGS WFS the detectors, that finally will be provided by ESO, ${ }^{13}$ have been considered as arrays of $240 \times 240 \mathrm{px}$ with $24 \mu \mathrm{m} / \mathrm{px}$ size. The main optical parameter of the LO and Ref WFS are resumed in Table 1 while Figure 12 shows the baseline optomechanical arrangement for the complete NGS WFS.

Table 1. Summary of the main optical parameters of the LO and Ref WFS.

\begin{tabular}{|l|c|c|}
\hline Parameter & LO-WFS & Ref-WFS \\
\hline N. of subapertures & $2 \times 2$ & $10 \times 10$ \\
Bandwidth & $1.5 \div 1.8 \mu \mathrm{m}$ & $0.6 \div 1.0 \mu \mathrm{m}$ \\
FoV & $1 \mathrm{arcsec}$ & $2 \mathrm{arcsec}$ \\
Pupil diameter & $5.76 \mathrm{~mm}$ & $5.76 \mathrm{~mm}$ \\
Sampling & $8.3 \mathrm{mas} / \mathrm{px}$ & $80.0 \mathrm{mas} / \mathrm{px}$ \\
Lenslet focal length & $87.7 \mathrm{~mm}$ & $8.8 \mathrm{~mm}$ \\
\hline
\end{tabular}

\section{CONCLUSIONS}

We presented in this paper the current status of the design of the MAORY NGS module at 6 months from the project preliminary design review. The design work has been carried out principally at the Arcetri Observatory. 

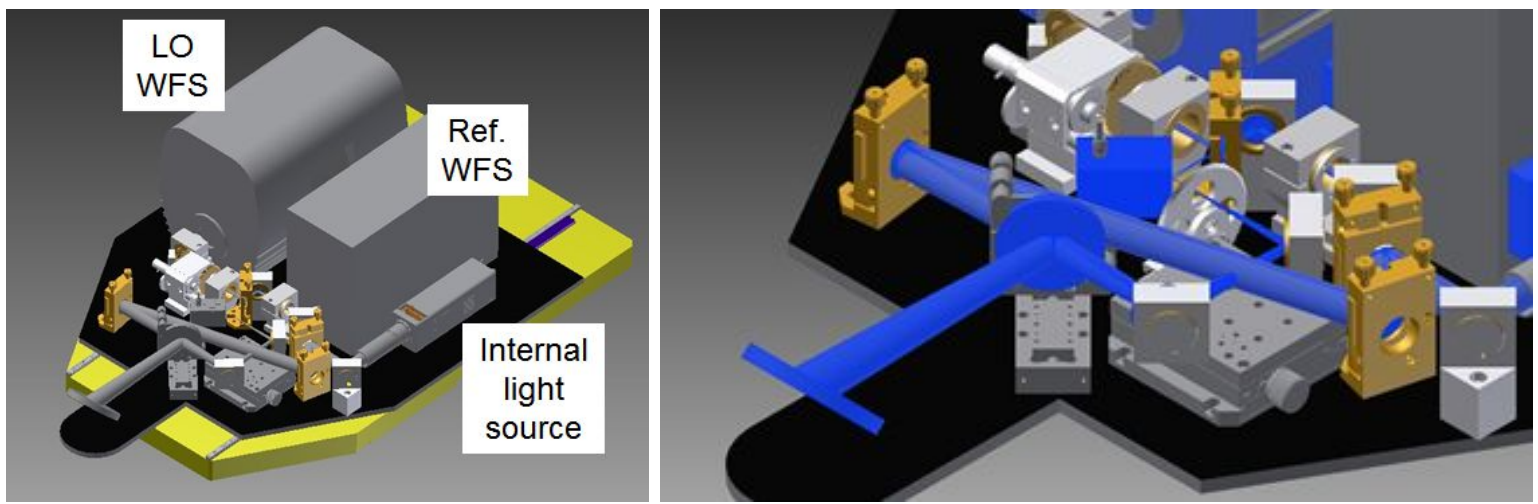

Figure 12. Left: model of the baseline optomechanical arrangement for the NGS WFS. Right: detail of the fore-optics optical path.

The technical requirements and functionalities for the NGS WFS have been identified. The baseline NGS WFS optomechanical arrangement presented here foresees the proper degrees of freedom to allow both the spectroscopic and the astrometric science programs of MICADO. The latest E2E simulations performed using the simulation tool PASSATA demonstrate that the baseline design of the NGS WFS will ensure the MAORY requirements for both sky coverage and limiting magnitude.

\section{REFERENCES}

1. E. Diolaiti, P. Ciliegi, et. al., MAORY: adaptive optics module for the E-ELT, Proc. SPIE 9909, 99092D (2016).

2. R. Davies, J. Schubert, et al., MICADO: first light imager for the E-ELT, Proc. SPIE 9908, 99081Z (2016).

3. R. Biasi, M. Manetti, M. Andrighettoni, et al., E-ELT M4 adaptive unit final design and construction: a progress report, Proc. SPIE 9909, 99097Y (2016).

4. M. Lombini, A. De Rosa, et al., Optical design of the post-focal relay of MAORY, Proc. SPIE 9908, 9908AB (2016).

5. L. Schreiber, E. Diolaiti, et al., Dimensioning the MAORY real time computer, Proc. SPIE 9909, 99094L (2016).

6. S. Esposito, G. Agapito, et al., NGS WFSs module for MAORY at E-ELT, Memorie della Societa Astronomica Italiana, v.86, p.446 (2015).

7. G. Agapito, A. Puglisi, S. Esposito, PASSATA: object oriented numerical simulation software for adaptive optics, Proc. SPIE 9909, 99097E (2016).

8. S. Oberti, MAORY design trade-off study: tomography dimensioning, Proc. from AO4ELT5 conference (2017).

9. L. Girardi, M. Barbieri, et al., TRILEGAL, a TRIdimensional modeL of thE GALaxy: Status and Future, Proc. of Astrophysics and Space Science, 26-165G (2012).

10. Y. Clenet, T. Buey, et al., Joint MICADO MAORY SCAO mode: specifications, prototyping, simulations and preliminary design, Proc. SPIE 9909, 99090A (2016).

11. H. Nicklas, et al., MICADO: the camera support structure at the E-ELT Nasmyth focus, Proc. SPIE 9908, 99089G (2016).

12. Electrical and Electronic Design Standards, GEN-SPE-ESO-50000-5401-4, Version 4.

13. M. Downing, M. Casali, et al., AO WFS detector developments at ESO to prepare for the E-ELT, Proc. SPIE 9909, 990914 (2016). 